\title{
Reliability and sources of control of preference for signaled shock
}

\author{
RALPH R. MILLER, NANCY A. MARLIN, and ALVIN M. BERK \\ Brooklyn College of the City University of New York, Brooklyn, New York 11210
}

Five experiments investigated the reliability of, and a possible explanation for, the preference for signaled shock (PSS) phenomenon, in which animals show a preference for signaled over unsignaled, unmodifiable shock. Experiment 1 demonstrated the generality of our previous PSS shuttlebox data. In an attempt to explain some of the studies in which a failure to obtain a PSS has been reported, Experiments 2 and 3 found that under certain conditions the PSS effect could be overridden by an innate preference for dark; however, the series of experiments in toto speaks clearly for the robustness of the phenomenon. Consistent with the recently proposed "crossover consequence hypothesis," Experiment 3 also revealed that shuttling behavior during manifestations of PSS is not randomly distributed in time. However, Experiments 4 and 5 found that PSS could not generally be attributed to an interaction between the acquired aversiveness of the signal and the spatially asymmetrical probability of shuttling being punished, as proposed by this hypothesis.

Since Wyckoff (1952) first introduced the concept of "observing behavior," researchers have been interested in why humans and animals seek predictive information about the occurrence of impending but unmodifiable reinforcers. Lockard's (1963) finding that rats prefer signaled over unsignaled unavoidable shock tentatively eliminated the possibility that secondary reinforcement could completely explain observing behavior. This conclusion was strengthened by a number of subsequent studies that effectively prevented the use of the signal to overtly reduce the intensity of the applied shock (e.g., Perkins, Seymann, Levis, \& Spencer, 1966). As a matter of potential theoretical importance, a large number of papers have appeared in recent years attempting to explain why animals prefer a signal prior to unmodifiable shock. At the same time, however, a few papers have appeared questioning the reliability of the effect. The present paper first addresses the issue of reliability and then turns to the possible sources of control of the preference for signaled shock (PSS) phenomenon.

\section{EXPERIMENT 1}

Biederman and Furedy have repeatedly (e.g., 1973) failed to observe the PSS effect when overt shock

This research was supported by National Science Foundation Grant BMS75-03383 and a grant from the Faculty Research Award Program of the City University of New York. R. R. M. is supported by National Institute of Mental Health Research Scientist Development Award MH-00061. Thanks are due David Daniel, Carla Fragomeni, and Michael Vigorito for their assistance in collecting the data and Joan Wessely for her aid in preparing the manuscript. Reprint requests should be addressed to Ralph R. Miller, Department of Psychology, Brooklyn College of the City University of New York, Brooklyn, New York 11210. modification was prohibited. Noting that those studies typically used fewer training trials than studies that obtained the effect, Miller, Daniel, and Berk (1974) performed a study similar to Biederman and Furedy's (1973), using fixed tail electrodes to deliver unmodifiable shock in a shuttlebox, and found a decided PSS after several hundred trials.

In a more recent critique of the PSS phenomenon, Biederman and Furedy (1976) have questioned the small number of subjects (three) used by Miller et al. (1974) while failing to note that each of these subjects met three successive independent criteria. In response to this criticism concerning group size, we feel that it is appropriate to briefly report a recent study that is a near replication of the experiment of Miller et al. (1974).

\section{Method}

Subjects and Apparatus. The subjects were eight naive male Sprague-Dawley-descended rats obtained from the Brooklyn College Breeding Colony at an approximate age of 75 days. All animals were individually housed and maintained on ad-lib water and $10 \mathrm{~g} /$ day of powdered Purina Lab Chow. This restricted diet helped prevent the subjects from outgrowing the apparatus.

The apparatus were four tilt-floor, two-compartment shuttleboxes, BRS-LVE Model 146-04, equipped with a dropped transparent Plexiglas ceiling $6 \mathrm{~cm}$ above the grid floor. Each ceiling contained a centered longitudinal slot through which the subject's tail was passed so that a pair of electrodes affixed to the tail were inaccessible to the rat's paws or mouth. The bottom $6 \mathrm{~cm}$ of one compartment wall was white and the other was black. Color was balanced in respect to left and right across boxes. Each apparatus was illuminated by an $8-W$ incandescent bulb $45 \mathrm{~cm}$ above the white compartments. For further details concerning the apparatus, see Miller et al. (1974).

Procedure. On each day of the experiment, every animal had tail electrodes affixed and was placed on the black side of the shuttlebox facing away from the white compartment. Each daily session lasted $90 \mathrm{~min}$ and was divided into 450 epochs of $12 \mathrm{sec}$ duration, with the subject's position being recorded at the start 
of each epoch. Days 1-4 were devoid of signal or shock and served to adapt the animals to the apparatus and determine initial position preferences. Starting on Day 5 , each animal received an .11-mA constant current, $60-\mathrm{Hz}$ sinusoidal tailshock of $.5-\mathrm{sec}$ duration initiated at the end of the fifth second of a random $25 \%$ of the epochs. If the animal was on one side of the shuttlebox (CS-US) at the start of a shock epoch, the shock was immediately preceded by a 5 -sec tone (approximately $2.9 \mathrm{kHz}$ ) of $76 \mathrm{~dB}\left(6 \mathrm{~dB}\right.$ above background, re:.0002 dynes $/ \mathrm{cm}^{2}$ ). If the animal was on the opposite side (US-CS), the same tone immediately followed the shock. The initial CS-US side was the least preferred side for each animal as determined on Days 3 and 4. Animals were run daily until they reached a preference criterion of spending at least $80 \%$ of the total epochs on one side for 3 consecutive days. To ensure that the preference data did not merely reflect perseveration on the side favored during adaptation, days were not counted toward criterion until animals sampled both contingencies by spending at least $20 \%$ of the total epochs on the nonpreferred side for 2 consecutive days. After reaching the 3-day criterion, each subject had a 4-day rest period, following which it was started again, in the same apparatus with the signal-shock relationships reversed. The same criterion was maintained, with spatial preference consistent with the last criterion reached being ignored until 2 consecutive days were achieved with less than $80 \%$ of the 450 epochs spent on that side. Upon reaching a second 3 -day $80 \%$ criterion, signal conditions were once again reversed and animals were run to a third criterion, again defined as 3 days on the same side on at least $80 \%$ of the epochs.

\section{Results and Discussion}

Five of the eight subjects completed the experimental requirements. All five of these animals displayed behavior consistent with a PSS and reached the first criterion in an average of 8.0 days, including the 3 criterion days. Upon reversal of conditions, four animals reversed their behavior and yielded a new PSS preference in an average of 16.5 days, the greater time being largely attributable to two animals perseverating strongly in their old preference and completely failing to sample the new CS-US condition, one for 15 days and the other for 23 days. The fifth animal yielded a preference for the US-CS condition in 6 days. Upon the second reversal of conditions, all five animals selected the CS-US condition in an average of 7.0 days. To ascertain if the subject that had shown a US-CS preference on the second criterion had an overriding position preference, its signal condition was reversed a third time and it was run to a fourth criterion. This time the subject switched sides and achieved the CS-US criterion in 9 days. Overall, the five rats reached 15 CS.US criteria and 1 US-CS criterion, $\chi^{2}(1)=10.56, p<.005$. The performance of a typical animal is illustrated in Figure 1 .

The three animals that failed to complete the study all achieved an initial criterion performance in an average of 9.0 days, with all subjects preferring the CS-US side. After conditions were reversed, all three subjects reachieved criterion performance in an average of 15.3 days, again with all choices being consistent with PSS. However, after the second reversal of conditions, these animals uniformly failed to achieve a third criterion before they were terminated due to irritation of the tail by the electrodes. Collectively, these three animals provide six further instances of PSS without the appearance of any contrary preferences.

\section{EXPERIMENT 2}

The observation in Experiment 1 of the PSS effect with tail electrodes and successive reversals, using parameters somewhat different from Miller et al. (1974), strengthened our confidence in the reliability of the effect. Consequently, we thought it appropriate to ask

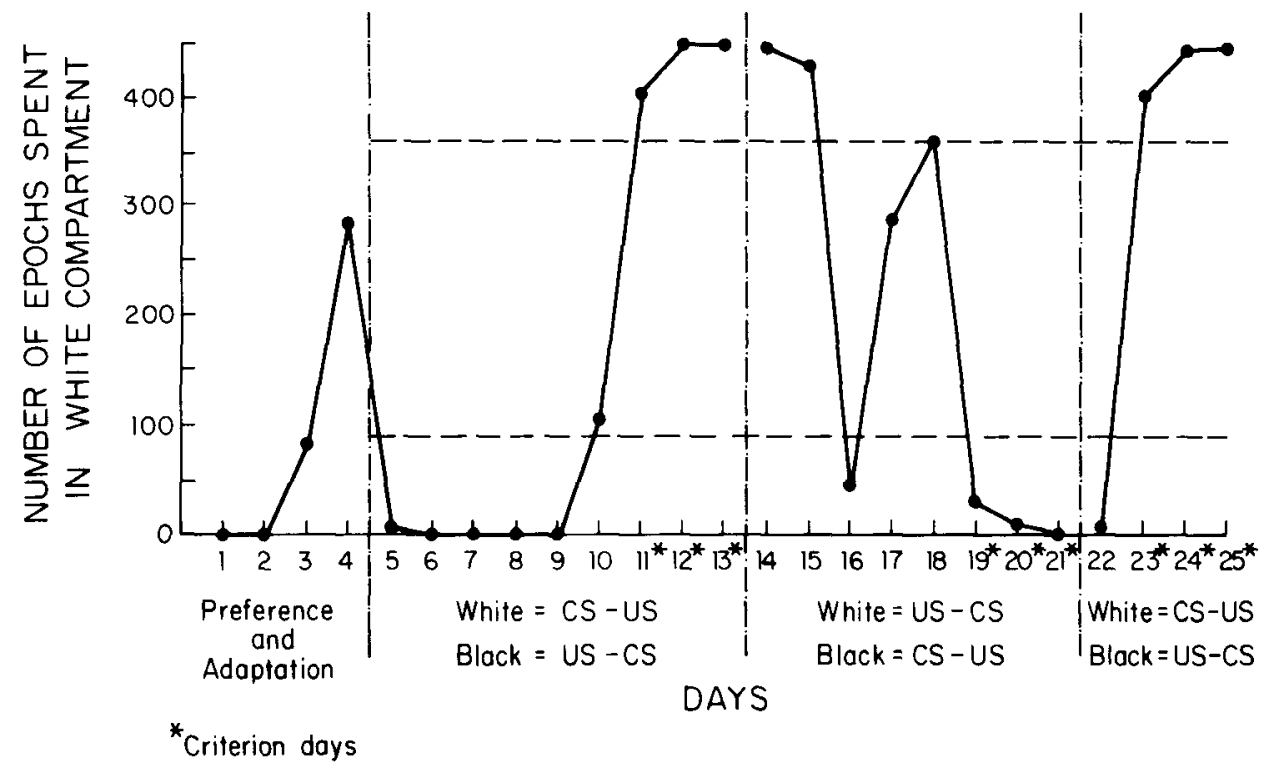

Figure 1. A representative subject in Experiment 1. There were 450 epochs per day. The dashed lines represent criterion values. The strong black compartment preference of Days 5-9 corresponded to the first 5 days of shock and probably was due to an innate dark preference aggravated by the stress of shock. 
why Furedy and his associates have had such difficulty obtaining the effect. Selecting Biederman and Furedy (1976) as representative, we noted that they increased the number of trials in that study from the low values of many of their earlier studies (e.g., Biederman \& Furedy, 1973) to a value in excess of that used in most of the reported instances of PSS. Yet a number of potential problems are evident: (1) Their .6-mA, 5.0-sec scrambled footshock [assumed to be unmodifiable based on data of Furedy and Biederman (1976)] was 10 times the duration and half the intensity used in most of the successful studies [e.g., Badia, Harsh, Coker, and Abbott (1976) used $1.6 \mathrm{~mA}$ for $.5 \mathrm{sec}$. (2) The signal duration was approximately half, or less, the duration used in the majority of successful studies, and its intensity (light and tone) were such as to suggest they may have been aversive, although critical background levels are not stated. (3) The correlated cues that the animals had available to aid them in differentiating the two compartments of the shuttlebox were black walls and white walls, which may have strongly affected the subjects' position preferences. The last possibility cannot be evaluated as Biederman and Furedy did not report behavior as a function of compartment color. However, it is noteworthy that Biederman and Furedy counterbalanced color across subjects for the signaled shock side and that many of their animals showed either a PSS or an inverse preference. Experiment 2 was designed to probe the effect upon PSS of using white and black walls as discriminative cues in conjunction with signaled and unsignaled footshock.

\section{Method}

Subjects and Apparatus. Twelve adult male Sprague-Dawleydescended rats (Charles River CD) were randomly assigned to one of three groups of four subjects each. The apparatuses were six shuttleboxes identical to the ones used in Experiment 1, except that the dropped ceilings were removed and replaced with 19-cm-high transparent ceilings and the tail electrodes were removed, as scrambled footshock rather than tailshock was used. The 5-cm-wide opening in the partition between the compartments was without a hurdle so as to avoid providing a shock-free ledge for the animals. Additionally, the previously 6-cm-high black and white strips on the walls of the shuttleboxes were replaced with strips $15 \mathrm{~cm}$ in height, the same height as that used by Biederman and Furedy (1976).

Procedure. On each of 14 consecutive days, the subjects were placed on the left side of the shuttlebox facing away from the divider and left for a $120-\mathrm{min}$ period that was divided into 480 epochs of $15-\mathrm{sec}$ duration. The position of each animal was recorded at the beginning of each epoch. On Day 1 , neither shock nor signal was presented. On Days 2-14, the subjects received .7-, 1.0-, or 1.4-mA constant current, scrambled, $60-\mathrm{Hz}$ footshocks (as a function of group assignment) for $.5 \mathrm{sec}$ on a random $20 \%$ of the epochs. Scrambled shock was provided by a $5,000-\mathrm{V}$ source driving a chain of NE-2 neon bulbs, one of which separated each pair of adjacent grids; this ensured that all possible pairs of grids were capable of delivering footshock. For half the subjects in each group, being on the white side caused the shock to be immediately preeeded by a $5-\mathrm{sec}, 24-\mathrm{Hz}$ click $5 \mathrm{~dB}$ above a white noise background of $67 \mathrm{~dB}$ (re: $.0002 \mathrm{dynes} / \mathrm{cm}^{2}$ ). If the animal was in the other compartment, the signal immediately followed the shock. For the other half of the subjects, the spatial conditions were reversed.

\section{Results and Discussion}

No differences in behavior as a function of shock intensity were observed. After the first three sessions, most subjects were individually consistent in their spatial preferences over days. The animals fell into three basic classes: (1) subjects displaying no appreciable spatial preference (two subjects); (2) subjects showing a strong preference by spending at least $80 \%$ of 3 consecutive days on the same side (CS-US $=2$, US-CS $=3$ ); and (3) subjects yielding a significant preference but not meeting the stringent criterion of (2) (CS-US $=4$, US-CS $=1$ ). However, this varied performance across animals as a function of signal condition was in sharp contrast to the finding that all 10 subjects displaying spatial preferences during the training sessions showed a preference for the black compartment, $\chi^{2}(1)=8.10$, $p<.01$. Of course, the possibility exists that the click signal was not discriminable to the animals, but the alerting response evoked by the click signal in the present experiment and the effectiveness of the sanie signal in Experiments 3, 4, and 5 reported below argue against this possibility.

Although Biederman and Furedy (1976) do not report data concerning preference as a function of compartment color, our data are consistent with theirs in yielding operationally defined preferences for either CS-US or US-CS conditions, but no uniform signal preference across animals. Notably, the PSS effect reported in Experiment 1 using a black-white discrimination was obtained with black and white surfaces only $6 \mathrm{~cm}$ high around the walls, whereas Biederman and Furedy (1976) and the present study used white and black walls $15 \mathrm{~cm}$ high. This difference may have changed the wall color from being merely a discriminative cue to being the focus of an innate dark preference exaggerated by the stress of shock. This hypothesized heightened dark preference could have overridden and obscured any possible PSS effect. However, it should be noted that in somewhat different situations other researchers as well as ourselves have obtained the PSS phenomenon with light vs. dark as the discriminative cue; the present failure should therefore be regarded as strongly parameterdependent.

\section{EXPERIMENT 3}

To test the possibility that a dark preference might have overshadowed a PSS, this experiment replicated Experiment 2 except for having discriminative cues equated for luminosity levels in the two compartments.

\section{Method}

Subjects and Apparatus. Another 12 adult male SpragueDawley-descended rats were randomly assigned to one of three groups of four subjects each. The apparatuses were identical to the shuttleboxes used in Experiment 2, except that the white walls were replaced with vertical .3-cm-wide alternating black and white stripes and the black walls with vertical $1.9-\mathrm{cm}$-wide alternating black and white stipes. To further equalize illumination levels, the 8-W chamber light in each apparatus was moved 
from above the previously white end of shuttlebox to an overhead point equidistant from the two compartments.

Procedure. As in Experiment 2, the subjects were placed in a shuttlebox each day for $48015-\mathrm{sec}$ epochs, with the position of every animal being recorded at the start of each epoch. In addition, shuttle responses of each subject were recorded. Neither shock nor signal was present on Day 1 . Thereafter, dependent upon group assignment, the subjects received .7-, 1.0-, or 1.4-mA, 5 -sec scrambled footshock on $20 \%$ of the epochs. For half the animals in each group, the narrow stripes corresponded to the CS-US condition and the wide stripes corresponded to the US-CS condition. For the other half of the subjects, the conditions were reversed. When possible, subjects were run to a second and third criterion identical to the first but with the added constraint that after a reversal of conditions, days with spatial preference consistent with the last criterion reached were ignored until 2 consecutive days were achieved with less than $80 \%$ of the 480 epochs spent on that side. Animals were run daily from Day 2 through Day 48 or until three consistent criteria had been reached, whichever came first.

\section{Results and Discussion}

Day 1 , which lacked signal and shock, yielded many shuttle responses per animal $(\overline{\mathrm{X}}=133)$ and no strong preference between the two compartments by any animal. No differences as a function of shock level were observed; consequently, further analysis pooled subjects over shock level. The 12 subjects reached a first criterion in an average of 10.2 days, including the 3 criterion days. Ten rats displayed a PSS and two yielded an inverse preference. This difference proved significant, $\chi^{2}(1)=$ $4.08, p<.05$. Over the course of the experiment, a total of 30 criteria were met by the 12 rats. Of these, 25 were consistent with PSS and 5 were inconsistent. This difference is highly significant, $\chi^{2}(1)=12.0$, $\mathrm{p}<.001$.

The two animals that initially made the inverse criterion subsequently made the criterion for PSS. However, two rats that initially made the PSS criterion then made the inverse criterion. This suggests that a position preference independent of the signal timing controlled the behavior of these four animals. However, two of these four animals subsequently switched their position preferences as control of the signal was spatially reversed, suggesting some complex relationship of signal preference, position preference independent of signal timing, and past experience.

In summary, this experiment demonstrates a robust but not universal PSS. These results stand in sharp contrast to Experiment 2, which differed only by not having equal luminosity in the two compartments. Collectively, Experiments 2 and 3 suggest one possible reason for the failure of Biederman and Furedy (1976) to observe a PSS.

Examining one other reported failure to obtain a PSS, Crabtree and Kruger (1975) used a shuttlebox rather similar to the one used in the present study. However, they used a shock duration four times that which has been used in most of the successful PSS studies. Perhaps more significantly, they gave each animal $3 \mathrm{~h}$ of exposure to the signal alone before the start of acquisi- tion. This may have induced a profound latent inhibition of associations to the signal.

\section{EXPERIMENT 4}

The traditionally cited sources of the PSS effect include: (1) preparatory responding (Perkins, 1968), (2) preference for signaled safety (Seligman, Maier, \& Solomon, 1971), and (3) information-seeking (Berlyne, 1960). In an elegant series of experiments, Badia and his colleagues (e.g., Badia, Harsh, Coker, \& Abbott, 1976; Freeman \& Badia, 1975) have established the importance of signaled safety in PSS, but not necessarily to the exclusion of other factors. In Experiment 1 and 3 of the present series, observation of the animals indicated that no overt postural modification occurred at the onset of, or during, the signal. However, in the CS-US condition, the animals in motion at the time of the signal onset did freeze until the initiation of shock. This suggests that if a preparatory response were being elicited by the signal, it would be internal in nature rather than overt (consistent with PSS being defined by the use of overtly unmodifiable shock).

Recently, Frankel and Vom Saal (1976) have suggested that much reported PSS behavior could be an artifact due to escape from the acquired aversiveness of the signal, an aversiveness which has previously been reported by Harsh and Badia (1974). Specifically, Frankel and Vom Saal believe that, in situations in which the alternative condition to signaled shock consists of a backwards (shock-signal) or random relationship between the signal and the shock, the subject may be changing to the siznaled shock condition in order to escape the aversive signal in the alternative condition. Conversely, while the signal is also aversive in the signaled shock condition, escape to the alternative condition is effectively punished by delivery of shock, thereby creating an asymmetry in shuttling likelihood that would yield an apparent PSS. Notably, the Frankel and Vom Saal hypothesis is applicable only in situations in which the animal's behavior affects the signal timing without appreciable delay, and it has no explanatory value in respect to the many PSS demonstrations using the "changeover" paradigm of Badia and his colleagues. Moreover, it is not relevant to situations lacking any signal in the alternative condition (although this design is open to other criticisms).

In Experiment 3, however, the animal's position did affect the signal immediately. The postshock signal was terminated if the subject crossed into the CS-US compartment. In addition to the animal's location, we also recorded the number of crossings during postshock signals (US-CS condition) and during equivalent postshock intervals with the signal absent (CS-US condition). Dividing by the number of full 5 -sec opportunities for such shuttling on criterion days, we found more postshock shuttling to occur during signals than in the 
absence of signals. This finding may be artificially inflated by subjects that shuttled more than once follow. ing a single shock, but aside from this qualification, it is consistent with the hypothesis and data of Frankel and Vom Saal.

To test the Frankel and Vom Saal hypothesis more directly, the ability of the subject to immediately affect the signal by shuttling was eliminated in this study.

\section{Method}

Subjects and Apparatus. The three animals in Experiment 3 that showed the strongest PSS after they had thrice reached our criterion of 3 successive days with more than $80 \%$ of their time on the same side were used as subjects. (Meeting the third criterion of Experiment 3 had taken these animals 14,23, and 24 days each, including 9 criterion days.) Each subject was run in the same apparatus as it experienced in Experiment 3. The control circuit was modified such that a shuttling response initiated during a signal or a shock did not affect stimulus presentations during that particular epoch.

Procedure. The subjects were then run exactly as in Experiment 3 until they had thrice more met the criterion defined in Experiment 3.

\section{Results and Discussion}

All three of the animals achieved the initial PSS criterion and two more PSS criteria following reversals of conditions without ever reaching the opposite criterion, $\chi^{2}(1)=7.1, p<.01$. In total, our three subjects took 16,24 , and 28 days (each including 9 criterion days) to reach the third criterion. This represents essentially no change in the number of days necessary for these animals to achieve the triple criteria in Experiment 3. Moreover, the rate of postshock shuttling was unchanged for these animals between the later parts of Experiment 3 and the present study.

Frankel and Vom Saal's hypothesis predicts that, without the ability to terminate the postshock signal by shuttling, the PSS effect we observed in Experiment 3 should have vanished in the present study, or at least should have diminished over sessions with increasing extinction of previously reinforced shuttling behavior. The observed stability of PSS in this experiment appears contrary to their hypothesis. However, it is possible that a preference established during Experiment 3 based on escape from the signal in the shocksignal condition could be very slow to extinguish.

\section{EXPERIMENT 5}

To further test the Frankel and Vom Saal hypothesis, we thought it appropriate to see if naive animals would exhibit a PSS without ever having been able to terminate the signal by shuttling.

\section{Method}

Subjects and Apparatus. Twelve naive adult male SpragueDawley-descended rats were assigned to one of two groups of six animals each. The apparatuses were identical to those used in Experiment 4, and the programming modification that prevented shuttling during signal or shock from having any effect during that epoch was maintained.
Procedure. One group was run daily for 480 epochs of 15 -sec duration with shock on a random $20 \%$ of the epochs. The other group was treated identically except that epoch duration was lengthened to $30 \mathrm{sec}$ in an effort to strengthen the observed PSS. This increased the daily session length trom 2 to $4 \mathrm{~h}$. All members of a group were run simultaneously. To avoid confounding epoch length with running order, the sequence in which the two groups were run was reversed every other day. As no differences as a function of shock intensity were observed in Experiment 4 , the present study used a .7-mA, .5 -sec shock for all animals. The $5-\mathrm{sec}$ signal was the same as in Experiments 2, 3, and 4. Day 1 consisted of adaptation to the apparatus with neither signal nor shock present. On Days 2-13, each subject had a choice between the signal-shock condition and the shock-signal condition. In this study, no criterion was used.

\section{Results and Discussion}

An Epoch Duration by Days analysis of variance yielded no difference between groups, $\mathrm{p}>.5$, or interaction, $\mathrm{p}>.1$; however, a decided effect of days was found, $F(11,110)=15.67, p<.01$. The basis for this effect can be seen in Figure 2 to be the acquisition of PSS over Days 2-5. A t test comparing the last 4 days' performance of each group against the random outcome of 240 epochs per day on the signal-shock side proved significant for both the 2 -h group, $t(5)=3.04, p<.05$, and the 4-h group, $t(5)=7.49, \mathrm{p}<.001$. Examination of individual animals showed that five out of six animals in each group displayed an overwhelming PSS that exceeded the previously used criterion of 3 consecutive days with $80 \%$ or more of the epochs on one side. The remaining animal in the 4-h group showed a strong PSS but failed to meet this criterion, and the remaining animal in the $2 \cdot h$ group displayed no preference at all.

This study clearly establishes that a robust PSS effect can be obtained in a shuttlebox without depend. ing upon asymmetrical consequences of shuttling during

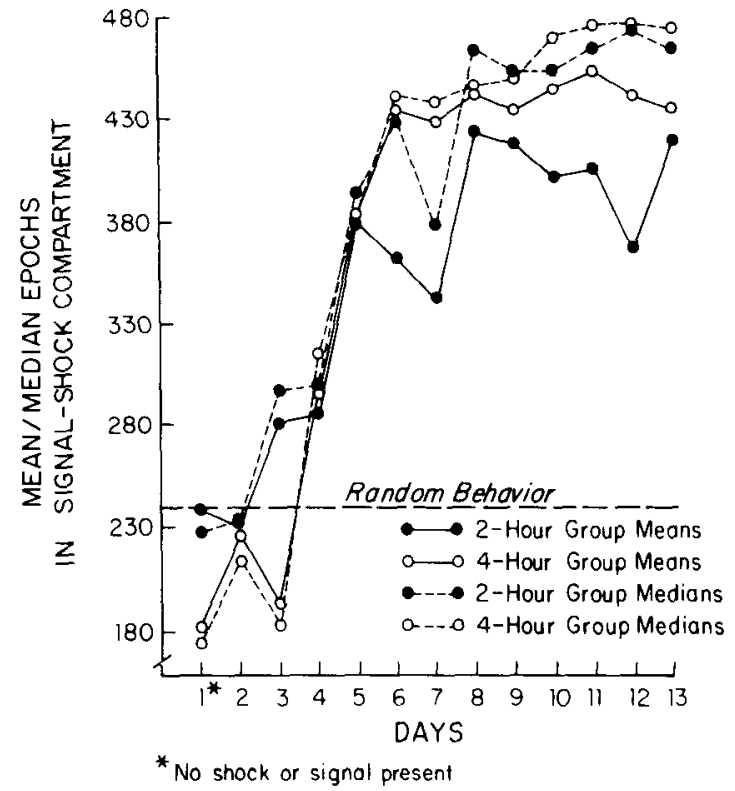

Figure 2. Group means and medians for Experiment 5. Values represent number of epochs out of $\mathbf{4 8 0}$ per day started on the side consistent with preference for signaled shock. 
the signal. This is contrary to the hypothesis of Frankel and Vom Saal (1976).

In summary, the PSS effect appears to be a readily obtainable phenomenon which is of special interest because of the theoretical implications of its viable proposed explanations. We are, however, impressed with and disturbed by two points. First, the effect is not universal across subjects under essentially identical conditions. And second, the effect did not appear until our animals received 200 to 300 shocks. Intuitively, this appears to be a large number of trials to learn a seemingly easy discrimination.

\section{REFERENCES}

Badia, P., Harsh, J., Coker, C. C., \& Abbott, B. Choice and the dependability of stimuli that predict shock and safety. Journal of the Experimental Analysis of Behavior, 1976, 26, 95-111.

Berlyne, D. E. Conflict, arousal, and curiosity. New York: McGraw-Hill, 1960.

Biederman, G. H., \& Furedy, J. J. Preference-for-signaledshock phenomenon: Effects of shock modifiability and light reinforcement. Journal of Experimental Psychology, 1973, 100, 380-386.

Biederman, G. B., \& Furedy, J. J. The preference-for-signaledshock phenomenon: Fifty days with scrambled shock in the shuttlebox. Bulletin of the Psychonomic Society, 1976, 7, 129-132.

Crabtree, M. S., \& Kruger, B. M. Free choice of signaled vs. unsignaled scrambled electric shock with rats. Bulletin of the Psychonomic Society, 1975, 6, 352-354.
Frankel, P. W., \& Vom SaAl, W. Preference for predicted over unpredicted shock. Quarterly Journal of Experimental Psychology, 1976, 28, 441-447.

FreEman, J., \& Badia, P. Do rats prefer information about shock intensity? Bulletin of the Psychonomic Society, 1975, 6, 75-78.

Furedy, J. J., \& Brederman, G. B. Preference for signaled shock phenomenon: Direct and indirect evidence for modifiability factors in the shuttlebox. Animal Learning \& Behavior, 1976, 4, $1-5$.

HARse, J., \& B BdiA, P. A concurrent assessment of the positive and negative properties of a signaled shock schedule. Animal Leaming \& Behavior, 1974, 2, 168-172.

LOCKARD, J. A. Choice of a warning signal or no warning signal in an unavoidable shock situation. Journal of Comparative and Physiological Psychology, 1963, 56, 526-530.

Miller, R. R., Daniel, D., \& Berk. A. M. Successive reversals of a discriminated preference for tailshock. Animal Learning \& Behavior, 1974, 2, 271-274.

Perkins, C. C., JR. An analysis of the concept of reinforcement. Psychological Review, 1968, 75, 155-172.

Perkins, C. C., Jr., Seymann, R. G., Levis, D. J., \& Spencer, H. R., JR. Factors affecting preference for signal-shock over shock-signal. Journal of Experimental Psychology, 1966, 72, 190-196.

Seligman, M. E. P., Maier, S. F., \& Solomon, R. L. Unpredictable and uncontrollable aversive events. In F. R. Brush (Ed.), Aversive conditioning and learning. New York: Academic Press, 1971. Pp. 347-400.

WyckofF, L. B. The role of observing responses in discrimination learning: Part 1. Psychological Review, 1952, 59, 431-442.

(Received for publication February 16, 1977; revision accepted May 12,1977.) 